\title{
Perfil clínico de la fibrilación auricular en el posoperatorio de cirugía cardíaca. Experiencia de un centro universitario
}

\author{
Dras. Jimena Pacella, Fernanda Molina, Andreina Gómez, Lucía Florio
}

\begin{abstract}
Resumen
Introducción: la fibrilación auricular (FA) es una complicación frecuente del posoperatorio de cirugía cardíaca (POCC). Internacionalmente se registra una incidencia de 30\%, siendo su pico máximo entre el segundo y tercer día de posoperatorio; nuestro objetivo fue conocer los datos en nuestro centro.

Método: se realizó un estudio de cohorte prospectivo, aprobado por el Comité de Ética institucional. Incluyó pacientes mayores de 18 años que recibieron cirugía cardíaca entre el $1^{\circ}$ de enero y el 31 de diciembre de 2018 en un centro cardiovascular universitario. Se excluyeron aquellos pacientes con FA al momento de la cirugía. Se registraron variables preoperatorias, operatorias y posoperatorias. Las variables continuas se presentaron como mediana e intervalo intercuartílico, las categóricas en valor absoluto y frecuencias relativas. La incidencia se calculó como número de casos nuevos/población total. Las asociaciones se evaluaron con chi cuadrado y Mann-Whitney test. Se realizó regresión logística univariada y multivariada. Se consideró significativo una $\mathrm{p}<0,05$.

Resultados: se incluyeron 104 pacientes. Mediana de edad 66 años; $51 \%$ sexo masculino. La incidencia de FA en el POCC (FAPO) fue de $29 \%$, con un máximo entre el segundo y tercer día, y una duración menor a 24 horas en $83 \%$ de los casos. La recurrencia durante el ingreso fue de 38\%. Se utilizó amiodarona para tratamiento agudo de la FAPO en el $100 \%$ de los casos, betabloqueantes en $63 \%$, digoxina en $7 \%$ y cardioversión eléctrica en $27 \%$. Todos los pacientes se encontraban en ritmo sinusal al alta, y al mes de la cirugía en los 20 casos en que se realizó electrocardiograma. Las variables con asociación significativa en análisis univariado para riesgo de FAPO fueron edad (OR 1,07, IC 95\%: 1,01-1,12 $\mathrm{p}=0,007$ ) y enfermedad renal crónica (OR 3,75, IC 95\%: 1,4-9,4 p=0,005). Un score de riesgo menor a 14 en el Multicenter Risk Index (OR 0,18, IC 95\%: 0,34-0,93 p=0,04) y el tabaquismo resultaron "protectores" en el análisis univariado (OR 0,38, IC 95\%: 0,14-0,98 p=0,048). En el análisis multivariado ninguna variable alcanzó significancia estadística. Se identificó asociación significativa entre FAPO y edema pulmonar $(\mathrm{p}<0,001)$, shock $(\mathrm{p}<0,04)$ e insuficiencia renal aguda ( $\mathrm{p}<0,01)$. Los pacientes con FAPO tuvieron una mediana de 5 días más de hospitalización $(\mathrm{p}<0,0003)$.

Conclusión: en la población estudiada se encontró una alta incidencia de FAPO. Se identificaron factores asociados a mayor riesgo de FAPO, así como asociación con otras complicaciones graves en el posoperatorio. Los resultados destacan la importancia de protocolizar el reconocimiento de los pacientes de riesgo así como su tratamiento.

Palabras clave: $\quad$ FIBRILACIÓN ATRIAL

ANTICOAGULANTES

CIRUGÍA CARDÍACA
\end{abstract}

\section{Clinical profile of postoperative atrial fibrillation in cardiac surgery; Experience of a university center}

\section{Summary}

Introduction: atrial fibrillation is a frequent postoperative complication of cardiac surgery. Internationally, an incidence of $30 \%$ is registered, being its maximum peak between the second and third postoperative day, however, we do not have data in our center.

Method: a prospective cohort study was carried out, approved by the institutional ethics committee. It included patients over the age of 18 years, who received cardiac surgery between January 1 and December 31, 2018 at a university cardiovascular center. Those patients with AF at the time of surgery were excluded. Preoperative, operative, and postoperative variables

Centro Cardiovascular Universitario, Hospital de Clínicas. Montevideo, Uruguay.

Correspondencia: Dra. Jimena Pacella. Correo electrónico: jimenapacella@gmail.com

Los autores declaran no tener conflictos de intereses.

$\mathrm{El}$ presente estudio no tiene fuentes de financiamiento.

Recibido May 6, 2020; aceptado Jul 8, 2020. 
were recorded. Continuous variables were presented as median and interquartile range, categorical variables in absolute value and relative frequencies. The incidence was calculated as the number of new cases / total population. Associations were evaluated with chi square and Mann Whitney test. Univariate and multivariate logistic regression was performed. A p $<0.05$ was considered significant.

Results: 104 patients were included. Median age of 66 years, $51 \%$ male. The incidence of postoperative atrial fibrillation was $29 \%$, with a maximum between the second and third day, and a duration of less than 24 hours in $83 \%$ of cases. Recurrence during admission was $38 \%$. Amiodarone was used for acute treatment of postoperative atrial fibrillation in $100 \%$ of cases, beta-blockers in 63\%, digoxin 7\%, and electrical cardioversion in 26\%. All the patients were in sinus rhythm at discharge and one month after surgery. Variables with a significant association in univariate analysis for postoperative atrial fibrillation risk were age (OR 1.07, 95\% CI: 1.01-1.12 $\mathrm{p}=0.007$ ) and chronic kidney disease (OR 3.75, 95\% CI: 1.4-9.4 p = 0.005). A risk score of less than 14 in the Multicenter Risk Index (OR 0.18; 95\% CI: 0.34-0.93 p = 0.04) and smoking were "protective" in the univariate analysis (OR 0.38; 95\% CI: $0.14-0.98 \mathrm{p}=0.048$ ). In the multivariate analysis, no variable reached statistical significance. A significant association was identified between postoperative atrial fibrillation and pulmonary edema $(p<0.001)$, shock $(\mathrm{p}<0.04)$ and acute renal failure $(\mathrm{p}<0.01)$. Postoperative atrial fibrillation patients had a median of 5 aditional days of hospitalization $(\mathrm{p}<0.0003)$.

Conclusion: A high incidence of postoperative atrial fibrillation was found in the studied population. Factors associated with a higher risk of postoperative atrial fibrillation were identified, as well as an association with other serious complications in the postoperative period. The results highlight the importance of protocolizing the recognition of risk patients as well as their treatment.

Key words:

ATRIAL FIBRILLATION

ANTICOAGULANTS

CARDIAC SURGERY

\section{Perfil clínico da fibrilação atrial no pós-operatório de cirurgia cardíaca; Experiência de um centro universitário}

\section{Resumo}

Introdução: a fibrilação atrial é uma complicação frequente no pós-operatório de cirurgia cardíaca. Internacionalmente, registra-se uma incidência de 30\%, sendo seu ponto máximo entre o segundo e o terceiro dia de pós-operatório, no entanto, não temos dados a respeito do nosso centro.

Método: foi realizado um estudo de coorte prospectivo, aprovado pelo comitê de ética institucional. O mesmo incluiu pacientes com idade superior a 18 anos, que receberam cirurgia cardíaca entre os dias 1ro de janeiro e 31 de dezembro de 2018 no centro cardiovascular universitário. Os pacientes com fibrilação atrial no momento da cirurgia foram excluídos. Foram registradas variáveis pré-operatórias, operatórias e pós-operatórias. As variáveis contínuas foram apresentadas em mediana e intervalo interquartil, as variáveis categóricas em valor absoluto e frequências relativas. A incidência foi calculada como o número de novos casos / população total. As associações foram avaliadas com o teste do qui quadrado e teste de Mann Whitney. Foi realizada a regressão logística univariada e multivariada. Foi considerado significativo um $\mathrm{p}<0,05$.

Resultados: 104 pacientes foram incluídos. Idade média de 66 anos, 51\% do sexo masculino. A incidência de fibrilação atrial no pós-operatório foi de 29\%, com um máximo entre o segundo e o terceiro dia,e uma duração inferior a 24 horas em $83 \%$ dos casos. A recorrência durante a internação foi de $38 \%$. A amiodarona foi utilizada no tratamento em agudo da fibrilação atrial no pós-operatório em $100 \%$ dos casos, betabloqueadores em 63\%, digoxina $7 \%$ e cardioversão elétrica em 26\%. Todos os pacientes estavam em ritmo sinusal no momento da alta e um mês após a cirurgia. As variáveis com associação significativa na análise univariada para risco de fibrilação atrial no pós-operatório foram: idade (OR 1,07, IC 95\%: 1,01-1,12 p = 0,007) e doença renal crônica (OR 3,75, IC 95\%: 1,4-9,4 p = 0,005). Um score de risco menor que 14 no Multicenter Risk Index (OR 0,18; IC 95\%: 0,34-0,93 p = 0,04) e o tabagismo foram "protetores" no univariado (OR 0,38; IC 95\%: 0,14-0,98 p = 0,048). Na análise multivariada, nenhuma variável atingiu significância estatística. Foi identificada associação significativa entre a fibrilação atrial no pós-operatório e edema pulmonar ( $p<0,001)$, choque cardiogênico $(p<0,04)$ e insuficiência renal aguda ( $p$ $<0,01$ ). Pacientes com fibrilação atrial no pós-operatório tiveram uma mediana de 5 dias a mais de internação $(\mathrm{p}<0,0003)$.

Conclusão: Foi encontrada alta incidência de fibrilação atrial no pós-operatório na população estudada. Foram identificados fatores associados a maior risco de fibrilação atrial no pós-operatório, bem como associação com outras complicações graves no pós-operatório. Os resultados destacam a importância de protocolar o reconhecimento de pacientes de risco e seu tratamento.

Palavras chave: $\quad$ FIBRILAÇÃO ATRIAL

ANTICOAGULANTES

CIRURGIA CARDÍACA 


\section{Introducción}

La fibrilación auricular (FA) en el posoperatorio de cirugía cardíaca (FAPO) es una complicación frecuente con incidencia cercana a $30 \%{ }^{(1)}$, con un máximo entre el segundo y tercer día de posoperatorio( ${ }^{(2)}$. Se ha identificado asociación entre FAPO y mayor morbilidad perioperatoria, mortalidad temprana y a largo plazo, así como un aumento de la estadía y los costos hospitalarios ${ }^{(3-5)}$.

En la mayoría de los casos la FAPO es autolimitada, con un período de predisposición a arritmias auriculares que se resuelve en las siguientes 6-12 semanas del posoperatorio(6).

La patogenia es multifactorial y los mecanismos fisiopatológicos asociados incluyen inflamación pericárdica, estrés oxidativo, disbalance autonómico, trauma auricular, y cambios en volumen y presión que se presentan durante el período posoperatorio $^{(7,8)}$.

Existen factores preoperatorios, operatorios y posoperatorios asociados a un mayor riesgo. La edad es el factor predisponente más consistente en los diferentes estudios. Cada diez años de aumento de la edad, se produce un aumento de $75 \%$ en la posibilidad de desarrollar $\mathrm{FA}^{(2)}$. La historia previa de FA, enfermedad pulmonar obstructiva crónica (EPOC), enfermedad renal crónica (ERC), obesidad, disfunción sistólica y diastólica ventricular, son otros de los factores de riesgo asociados. De los factores intraoperatorios, la cirugía valvular es uno de los más importantes.

La suspensión de la terapia betabloqueante (BB) en el posoperatorio inmediato resulta en riesgo aumentado de desarrollar $\mathrm{FA}^{(9,10)}$. Cuando los $\mathrm{BB}$ son administrados en el pre y posoperatorio o incluso iniciados en el posoperatorio inmediato, reducen de forma significativa el riesgo de $\mathrm{FAPO}^{(2)}$. De forma similar el inicio de la terapia con inhibidores de la enzima conversora de angiotensina (IECA) antes y después de la cirugía se asocia a un menor riesgo de FAPO ${ }^{(11)}$. Existen en la literatura scores de riesgo, como el Multicenter Risk Index for Atrial Fibrilation After Cardiac Surgery, que permite identificar pacientes con mayor riesgo de $\mathrm{FAPO}^{(2)}$.Un puntaje menor a 14 fue considerado de bajo riesgo, entre 14-31 de moderado riesgo, y más de 31 de alto riesgo de FAPO.

Los objetivos de tratamiento de la FAPO son similares a los de la FA en otro contexto clínico, incluyendo la prevención de eventos tromboembólicos, control de la frecuencia ventricular, cardioversión y mantenimiento del ritmo sinusal. Gillinov y colaboradores, autores del Rate Control versus Rhythm Control for Atrial Fibrillation after Cardiac Surgery, publicado en NEJM en mayo de 2016, conclu-
Principales aportes

- Primer estudio de FA en posoperatorio de cirugía cardíaca en nuestro centro.

- Los resultados obtenidos son comparables a reportes internacionales.

- El estudio puede conducir a la protocolización de la actuación en la FA en el posoperatorio de cirugía cardíaca.

yeron que las estrategias de control de frecuencia y ritmo en la FAPO fueron asociadas a similares días de hospitalización, tasa de complicaciones y similar baja tasa de persistencia de FA a los 60 días ${ }^{(12-16)}$. El beneficio de la anticoagulación precoz para la profilaxis del ataque cerebrovascular (ACV) en la FAPO es incierto, dado que puede verse como una respuesta transitoria al estrés fisiológico. En algunos estudios la FAPO se asocia a aumento del riesgo de ACV temprano y morbimortalidad a los 30 días. Gialdini y colaboradores demostraron que este aumento era mayor en caso de FA vinculada a cirugía no cardíaca ${ }^{(17,18)}$. La guía de práctica de la Sociedad Europea de Cardiología (ESC) de 2016 sobre diagnóstico y tratamiento de la FA establece que se debe considerar la anticoagulación a largo plazo de los pacientes con FA y riesgo de ACV tras la cirugía cardíaca, teniendo en cuenta el riesgo individual de ACV y sangrado (según los scores $\mathrm{CHA}_{2} \mathrm{DS}_{2}$-VASc y HAS-BLED) ${ }^{(15)}$.

Sin embargo, la información con la que contamos surge de estudios y evidencia internacionales, no existiendo la información sobre la población de nuestro país, o siendo esta muy escasa.

\section{Objetivos}

\section{Primario}

- Determinar la incidencia de FAPO en el Centro Cardiovascular Universitario (CCVU) del Hospital de Clínicas.

\section{Secundarios}

- Identificar factores predisponentes para el desarrollo de FAPO.

- Aplicar el índice de riesgo Multicenter Risk Index for Atrial Fibrillation After Cardiac Surgery en una población uruguaya.

- Determinar si existe asociación entre FAPO y complicaciones en el posoperatorio de cirugía cardíaca.

- Describir el tratamiento utilizado para la FAPO en el CCVU. 
- Evaluar el ritmo cardíaco en el primer control en policlínica, al mes de la cirugía.

\section{Material y método}

Estudio de cohorte prospectivo que incluyó a pacientes de ambos sexos, mayores de 18 años, que recibieron cirugía cardíaca en el CCVU del Hospital de Clínicas en el período comprendido entre el $1^{\circ} \mathrm{de}$ enero y 31 de diciembre de 2018. Se excluyeron de este estudio aquellos pacientes que se encontraban en FA al momento de la cirugía.

Los datos clínicos se obtuvieron a través de la historia clínica, incluyendo variables preoperatorias, operatorias y posoperatorias.

Las variables preoperatorias fueron: sexo, edad, índice de masa corporal (IMC), historia previa de hipertensión arterial (HTA), diabetes mellitus (DM), dislipemia, tabaquismo, EPOC, ERC, historia de FA paroxística previa documentada y ACV. Tratamiento previo a la cirugía con IECA, antagonistas del receptor de angiotensina II (ARA II), BB, antagonistas del calcio (AC) y estatinas. De las variables ecocardiográficas se registraron la fracción de eyección del ventrículo izquierdo (FEVI), disfunción diastólica y tamaño de aurícula izquierda.

De la paraclínica preoperatoria se registró el clearance de creatinina y la hemoglobinemia.

A todos los pacientes se les realizó el cálculo de riesgo de $\mathrm{ACV}$ utilizando el score $\mathrm{CHA}_{2} \mathrm{DS}_{2}$-VASc y a quienes se les realizó cirugía de revascularización miocárdica también se les aplicó el Multicenter Risk Index for Atrial Fibrillation After Cardiac Surgery $^{(2)}$ para evaluar riesgo de desarrollo de FA. Se consideró un puntaje menor a 14 como de bajo riesgo, entre 14-31 de moderado riesgo y mayor de 31 de alto riesgo.

De las variables operatorias se tomaron en cuenta la oportunidad de la cirugía (urgencia o coordinación), tipo de cirugía (valvular, coronaria o combinada), uso y tiempo de circulación extracorpórea (CEC) y tiempo de clampeo aórtico (CA). En el posoperatorio se analizaron el tiempo de requerimiento de intubación orotraqueal (IOT), requerimiento y duración del uso de vasopresores. Al momento del egreso de cuidados intensivos e ingreso a la unidad cardiológica se registró si recibían o no tratamiento con IECA o ARAII, y BB o calcio-antagonistas..

Se definió FAPO como la ausencia de onda $\mathrm{P}$ antes del complejo QRS, junto a un ritmo irregular ventricular identificado mediante electrocardiograma o monitorización electrocardiográfica con una duración $\geq 5$ min durante la estancia hospitalaria. No se consideró FAPO si ocurría luego del alta.
Una vez que el paciente presentaba FA, se determinó el día de presentación en el posoperatorio, duración, tratamiento recibido y recurrencia durante el ingreso luego de un período en ritmo sinusal. Dentro de las complicaciones posoperatorias analizadas se incluyeron: muerte, infecciones, reoperación, edema pulmonar, shock, insuficiencia renal aguda (aumento de la creatininemia $\geq 0,5 \mathrm{mg} / \mathrm{dl}$ ), ACV o ataque isquémico transitorio (confirmado por tomografía computada), infarto agudo de miocardio (IAM) posoperatorio (según la cuarta definición universal de infarto)(19), derrame pericárdico con requerimiento de drenaje, bloqueo auriculoventricular completo (BAVC) y reingreso a los 30 días. Se determinó la duración de la internación y ritmo cardíaco al alta en todos los pacientes.

En quienes habían presentado FA, se registró tratamiento con amiodarona y anticoagulación al alta, así como ritmo cardíaco al mes de posoperatorio durante el primer control en policlínica.

\section{Análisis estadístico}

El análisis estadístico se realizó utilizando el sofware STATA ${ }^{\circledR} 13$.

Las variables continuas se presentaron en forma de mediana e intervalo intercuartílico, mientras que las variables categóricas se expresaron mediante su valor absoluto y frecuencias relativas.

La incidencia de FA se calculó como el número de casos nuevos sobre el total de la población.

Para las variables categóricas se utilizó el test $\chi^{2}$ para evaluar asociaciones, mientras que las comparaciones de variables continuas se realizaron con Mann-Whitney (test no paramétrico). Para identificar variables de riesgo se realizó una regresión logística univariada y multivariada definiendo el odds ratio (OR). Se consideró significativo un valor $\mathrm{p}<0,05$.

El estudio respetó los criterios establecidos en la Declaración de Helsinki y fue aprobado por el Comité de Ética de la Investigación del Hospital de Clínicas.

\section{Resultados}

En el período estudiado se realizaron un total de 110 cirugías cardíacas en el CCVU del Hospital de Clínicas (seis de los pacientes cumplían criterios de exclusión), incluyéndose finalmente 104 pacientes.

La mediana de edad fue de 66 años, aproximadamente la mitad de los pacientes eran de sexo masculino $(51 \%)$. La mayoría eran hipertensos $(72 \%)$ y solo $4 \%$ presentaba FA previa. El resto de las características de la población se presenta en la tabla 1.

Con respecto a las características operatorias (tabla 2), el 56\% de las cirugías fueron de coordina- 
ción. La mayoría (68\%) correspondió a revascularización miocárdica, seguida de sustitución valvular aórtica aislada (18\%), coronario-aórtica (6\%), mitral aislada (4\%) y coronario-mitral (4\%).

En el 85\% de los procedimientos se utilizó CEC. La mediana de tiempo de CEC fue de 90 minutos, y 57 minutos la mediana del CA.

La incidencia de FAPO fue de 29\% (30 pacientes), siendo más frecuente en los primeros cinco días de posoperatorio, con una incidencia máxima entre el segundo y tercer día (figura 1). En la mayoría de los casos (83\%), la FA duró menos de 24 horas, con un porcentaje de recurrencia de $38 \%$ durante la internación, mayor entre el tercer y quinto día (figuras 2 y 3 ). Todos los pacientes con FAPO recibieron amiodarona durante la internación, 63\% (19) $\mathrm{BB}, 7 \%$ (2) digoxina y $27 \%$ (8) requirieron cardioversión eléctrica. Del total de pacientes con FAPO, $56 \%$ (17) fue anticoagulado durante la internación (figura 4). Al momento del alta, todos los pacientes se encontraban en ritmo sinusal, 63\% (19) recibió amiodarona y $30 \%$ (9) fue anticoagulado (figura 5). Se registró el ritmo cardíaco al mes de la cirugía en 20 de los 30 pacientes que tuvieron FAPO, estando todos en ritmo sinusal. Diez de los 30 pacientes se perdieron en el seguimiento.

En el análisis univariado las variables que mostraron asociación significativa con el riesgo de FAPO fueron la edad (OR 1,07, IC 95\%: 1,01-1,12; $\mathrm{p}=0,007$ ) y la presencia de ERC (OR 3,75, IC 95\%: 1,4-9,4 $\mathrm{p}=0,005)$. Se identificaron como factores protectores para el desarrollo de FAPO un score de riesgo bajo para FA (OR 0,18, IC 95\%: 0,34-0,93 $\mathrm{p}=0,04)$ y el consumo actual de tabaco (OR 0,38 , IC 95\%: 0,14-0,98 p=0,048) (tabla 3).

En el análisis multivariado ninguna de las variables alcanzó significancia estadística (tabla 4).

En cuanto a las complicaciones posoperatorias, se identificó una asociación estadísticamente significativa entre la presencia de FAPO y desarrollo de edema pulmonar $(p<0,001)$, shock $(p=0,04)$ e insuficiencia renal aguda $(p=0,01)$. La mediana de duración de internación en los pacientes que tuvieron FA fue de 12 días, mientras que en los que no presentaron FA fue de 7 días, siendo esta diferencia estadísticamente significativa $(p=0,0003)$ (tabla 5).

\section{Discusión}

Hasta el momento no teníamos información sobre las características de la FAPO en nuestro centro. Como antecedente de estudios sobre FAPO a nivel nacional se encuentra el publicado en 2007 por Irigoin y colaboradores ${ }^{(19)}$, cuyo objetivo fue determinar la incidencia de FAPO de cirugía de revasculari-
Tabla 1. Características basales de la población .

$\mathrm{N}$ total $=104$

Sexo masculino - FR (n)

$0,51(53)$

Edad en años- mediana e IQ

$66(57-72)$

IMC - FR (n)

$<$ de 25

$0,39(41)$

$25-30$

$0,27(28)$

$>$ de 30

$0,34(35)$

Clearance de creatinina mediana $75(62-88)$

e IQ $\left(\mathrm{ml} / \mathrm{min} / 1,73 \mathrm{~m}^{2}\right)$

Enfermedad renal crónica - FR (n)

$0,27(28)$

Hipertensión arterial - FR (n)

$0,72(75)$

Diabetes mellitus - FR (n)

$0,38(39)$

ACV previo - FR (n)

$0,08(9)$

Tabaquismo - FR (n)

$0,38(40)$

EPOC - FR (n)

$0,11(11)$

FA previa - FR (n)

$0,05(5)$

$\mathrm{Hb}(\mathrm{mg} / \mathrm{dl})$ - mediana e IQ

$12,7(11,4-13,9)$

FEVI (\%) - mediana e IQ

$55(44,5-60)$

Disfunción diastólica - FR (n)

$0,45(47)$

Auriculomegalia izquierda - FR (n)

Leve

$0,24(25)$

Moderada

0,13 (14)

Severa

$0,11(11)$

IECA o ARA II - FR (n)

$0,63(66)$

$\mathrm{BB}$ o AC - FR (n)

$0,59(61)$

Estatinas - FR (n) $0,43(45)$

$\mathrm{CHA}_{2} \mathrm{DS}_{2}$-VASc FR (n)

$0,04(4)$

1

$0,12(12)$

2

$0,29(30)$

3

$0,29(30)$

$0,13(13)$

$0,08(8)$

$0,04(4)$

6

$0,02(2)$

Score de riesgo para FA FR (n)

Bajo

Moderado

$0,8(40)$

Alto

$0,13(14)$

Valores expresados en frecuencia relativa, mediana e intervalo intercuartilico.

IMC: índice de masa corporal; ACV: ataque cerebrovascular; $\mathrm{Hb}$ : hemoglobina; EPOC: enfermedad pulmonar obstructiva crónica; FA: fibrilación auricular; FEVI: fracción de eyección del ventrículo izquierdo; IECA: inhibidores de la enzima conversora de angiotensina; $\mathrm{BB}$ : betabloqueante; $\mathrm{AC}$ : antagonistas del calcio; FR: frecuencia relativa.

Disfunción diastólica definida como relación $\mathrm{E} / \mathrm{e}^{\prime}>14$; auriculomegalia izquierda determinada por volumen indexado; IQ: intervalo intercuartílico. 


\begin{tabular}{|c|c|}
\hline Oportunidad & $\mathrm{FR}(n)$ \\
\hline Urgencia & $0,44(46)$ \\
\hline Coordinada & $0,56(58)$ \\
\hline Tipo de cirugía & FR (n) \\
\hline Coronaria & $0,68(71)$ \\
\hline Valvular aórtica & $0,18(19)$ \\
\hline Coronario-aórtica & $0,06(6)$ \\
\hline Valvular mitral & $0,04(4)$ \\
\hline Coronario-mitral & $0,04(4)$ \\
\hline Con CEC & $0,85(88)$ \\
\hline Sin CEC & $0,15(16)$ \\
\hline $\begin{array}{l}\text { Tiempo de CEC (minutos) - } \\
\text { mediana e IQ }\end{array}$ & $90(72-119)$ \\
\hline $\begin{array}{l}\text { Tiempo de CA (minutos) - } \\
\text { mediana e IQ }\end{array}$ & $57(43-78)$ \\
\hline
\end{tabular}

zación miocárdica estratificada según el uso o no de CEC. Se incluyó a un total de 2459 cirugías entre los años 1994 y 2005. Los autores concluyeron que el uso de CEC se asoció con mayor incidencia de diagnóstico de FAPO, aunque con escaso significado clínico. En relación a nuestro estudio no se encontró asociación estadísticamente significativa entre el uso de CEC y desarrollo de FAPO. En nuestro centro, la mayoría de las cirugías realizadas son revascularizaciones coronarias utilizando CEC.

La incidencia encontrada de FAPO fue de $29 \%$, la mayoría de los casos se observaron entre el segundo y tercer día de posoperatorio, con una tasa de recurrencia cercana a $40 \%$, lo que coincide con lo reportado internacionalmente ${ }^{(1)}$. Los pacientes con más riesgo de presentar FAPO son los más añosos (cada año aumenta 1,07 veces la posibilidad de FA), antecedente de ERC y aquellos que recibieron cirugía combinada coronario-valvular. Ninguna variable mostró significancia estadística en el análisis multivariado, esto puede deberse al bajo número de pacientes del estudio.

Las estrategias de manejo de la FAPO en nuestro estudio fueron consistentes con la práctica clínica general, donde se suele optar por tratamiento médico con alta tasa de reversión a ritmo sinusal a corto plazo. La amiodarona y los BB fueron los fármacos más utilizados, requiriendo cardioversión eléctrica en ocho pacientes. Apenas el 30\% de los pa-

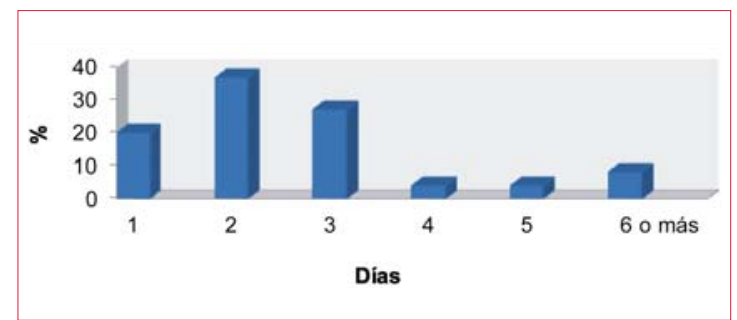

Figura 1. Día de posoperatorio de desarrollo de FA.

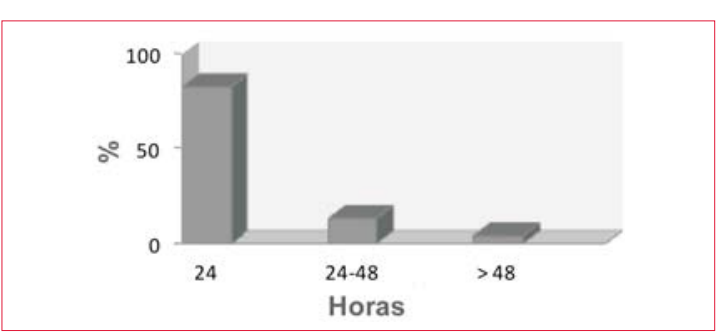

Figura 2. Duración de la FA.

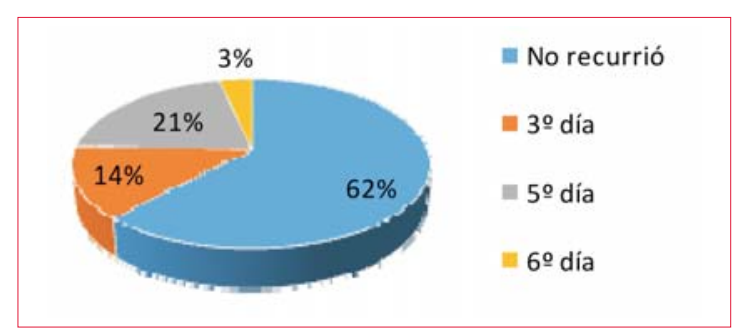

Figura 3. Recurrencia.

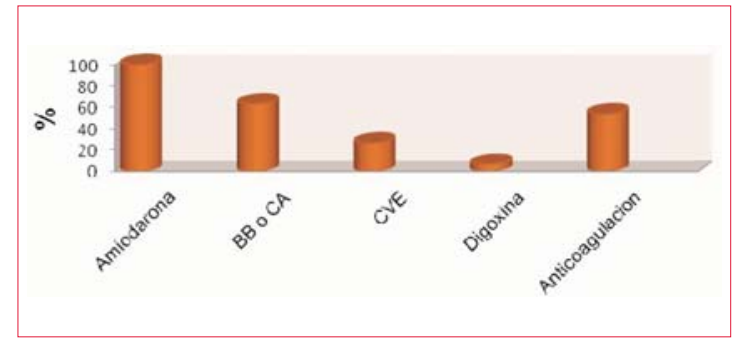

Figura 4. Tratamiento agudo de la FA. BB: betabloqueantes; CA: calcio-antagonistas; CVE: cardioversión eléctrica.

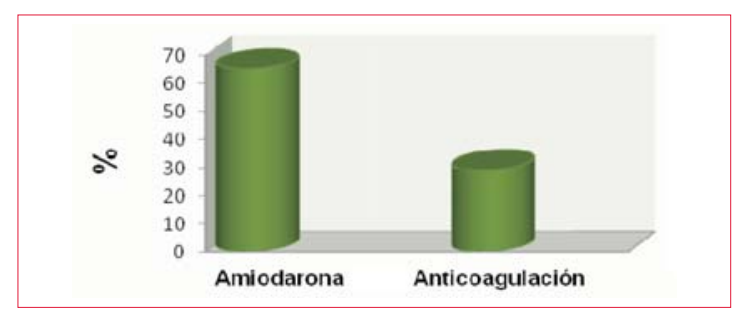

Figura 5. Tratamiento al alta.

cientes que presentaron FA fueron dados de alta con tratamiento anticoagulante, lo que es un bajo porcentaje, considerando que el $80 \%$ de nuestra población presentaba un puntaje $\mathrm{CHA}_{2} \mathrm{DS}_{2}$-VASc $\geq 2$. 
Tabla 3. Análisis de regresión logística univariado para factores predictores de FA preoperatorios, operatorios y posoperatorios.

\begin{tabular}{|c|c|c|c|}
\hline Variables & Odds ratio & IC $95 \%$ & Valorp \\
\hline Sexo masculino & 1,1 & $0,48-2,6$ & 0,75 \\
\hline Edad (c/año) & 1,07 & $1,01-1,12$ & 0,007 \\
\hline HTA & 0,69 & $0,27-1,73$ & 0,43 \\
\hline $\mathrm{DM}$ & 1,1 & $0,48-2,7$ & 0,73 \\
\hline Tabaquismo & 0,37 & $0,14-0,98$ & 0,04 \\
\hline EPOC & 1,4 & $0,39-5,3$ & 0,57 \\
\hline FA previa & 4 & $0,63-25$ & 0,14 \\
\hline ACV previo & 2,1 & $0,52-8,5$ & 0,28 \\
\hline Dislipemia & 0,67 & $0,24-1,8$ & 0,45 \\
\hline IECA o ARA II (preoperatorio) & 1,2 & $0,49-2,9$ & 0,66 \\
\hline BB o AC (preoperatorio) & 1,9 & $0,80-4,9$ & 0,13 \\
\hline Estatinas (preoperatorio) & 1,4 & $0,62-3,4$ & 0,37 \\
\hline FEVI & 0,98 & $0,95-1,01$ & 0,27 \\
\hline Disfunción diastólica & 1,5 & $0,67-3,7$ & 0,29 \\
\hline Clearance de creatinina & 0,97 & $0,98-0,99$ & 0,007 \\
\hline Enfermedad renal crónica & 3,75 & $1,4-9,4$ & 0,005 \\
\hline \multicolumn{4}{|l|}{ Score de riesgo } \\
\hline Bajo & 0,18 & $0,34-0,93$ & 0,041 \\
\hline Moderado & 0,6 & $0,20-1,72$ & 0,34 \\
\hline Alto & 2,4 & $0,64-8,9$ & 0,19 \\
\hline Cirugía de urgencia & 0,52 & $0,21-1,27$ & 0,15 \\
\hline Cirugía coronaria & 0,48 & $0,19-1,17$ & 0,10 \\
\hline Cirugía aórtica & 1,15 & $0,39-3,38$ & 0,79 \\
\hline Cirugía mitral & 2,5 & $0,34-18$ & 0,36 \\
\hline Cirugía coronaria-aórtica & 5,4 & 0,94-31 & 0,05 \\
\hline Cirugía coronario-mitral & 0,59 & $0,63-5,5$ & 0,64 \\
\hline Cirugía con CEC & 1,91 & $0,50-7,2$ & 0,33 \\
\hline Tiempo de clampeo & 1,0 & $0,99-1,0$ & 0,12 \\
\hline Tiempo de CEC & 1,0 & $0,99-1,01$ & 0,10 \\
\hline \multicolumn{4}{|c|}{$\begin{array}{l}\text { Disfunción diastólica definida como relación E/e'> 14. HTA: hipertensión arterial; DM: diabetes mellitus; EPOC: enfermedad pul- } \\
\text { monar obstructiva crónica; FA: fibrilación auricular; ACV: ataque cerebrovascular; IECA: inhibidores de la enzima conversora de la } \\
\text { angiotensina; ARA II: antagonistas del receptor de angiotensina II; BB: betabloqueantes; AC: antagonistas de calcio; FEVI: fracción } \\
\text { de eyección del ventrículo izquierdo. }\end{array}$} \\
\hline
\end{tabular}

En cuanto a las complicaciones, varios estudios han demostrado que la FAPO se asocia a un aumento en la morbimortalidad. En nuestro caso, la mortalidad registrada fue de $7 \%$, no habiendo encontrado asociación estadísticamente significativa con FAPO. Sin embargo, se encontró asociación signifi- cativa entre FAPO y complicaciones graves como shock, edema pulmonar e insuficiencia renal aguda. No podemos definir si es la FA la causante de dichas complicaciones o viceversa, pero sí podemos afirmar que existe una fuerte asociación entre ellas. 


\begin{tabular}{|c|c|c|c|}
\hline Variables & Odds ratio & IC $95 \%$ & Valorp \\
\hline Edad & 1,03 & $0,96-1,11$ & 0,81 \\
\hline Tabaquismo & 0,49 & $0,14-1,7$ & 0,26 \\
\hline Clearance de creatinina & 0,99 & $0,95-1,03$ & 0,84 \\
\hline Enfermedad renal crónica & 1,8 & $0,30-11,4$ & 0,26 \\
\hline \multicolumn{4}{|l|}{ Score de riesgo } \\
\hline Bajo & 0,22 & $0,34-1,4$ & 0,11 \\
\hline Moderado & 0,43 & $0,12-1,4$ & 0,16 \\
\hline Alto & 1,7 & $0,30-9,8$ & 0,53 \\
\hline Cirugía coronaria-aórtica & 1,34 & $0,11-16$ & 0,81 \\
\hline
\end{tabular}

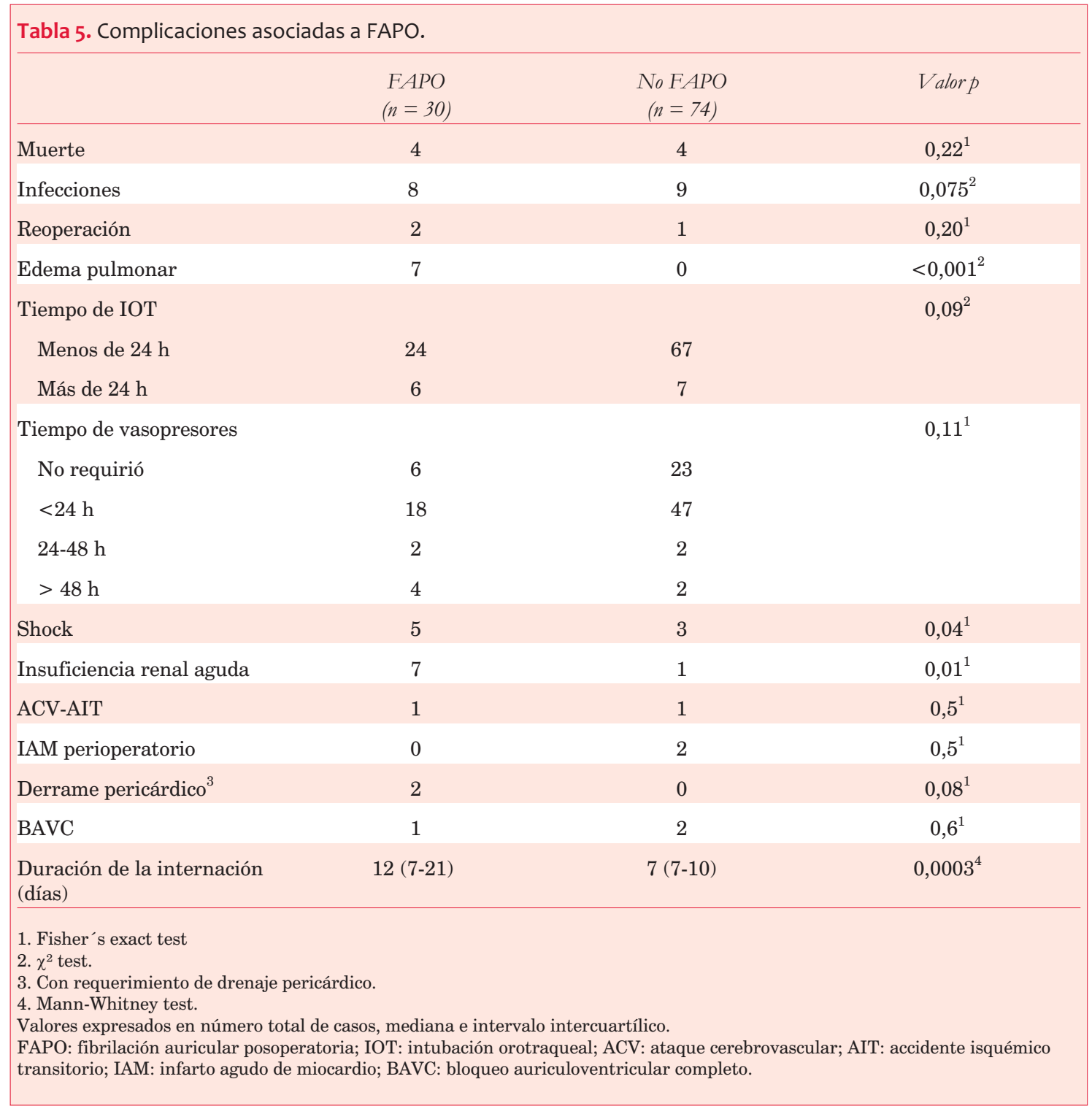


En concordancia con el resto de la evidencia existente, los pacientes que presentaron FAPO tuvieron una estancia hospitalaria más prolongada, lo que implica mayores gastos hospitalarios.

La FAPO es considerada en la mayoría de los casos autolimitada. A favor de esto, en nuestro caso, encontramos que todos los pacientes que tuvieron FA durante el posoperatorio se encontraban en ritmo sinusal al momento del alta y en los 20 pacientes que fueron evaluados al mes del procedimiento quirúrgico.

\section{Limitaciones}

Son limitaciones de nuestro trabajo el bajo número de pacientes y el hecho de tratarse de un estudio unicéntrico con baja capacidad de extrapolación de resultados. Dado que el seguimiento fue durante la internación y luego al mes en el control en policlínica, desconocemos el comportamiento de la FA a largo plazo en estos pacientes. Las variables del estudio se recolectaron retrospectivamente desde las historias clínicas, por lo que podría haber un subregistro de estas.

\section{Conclusiones y perspectivas}

A partir de este estudio conocemos la incidencia, los factores de riesgo predisponentes y las complicaciones de la FAPO en nuestro centro, siendo cualitativamente comparables a lo reportado internacionalmente.

La FAPO se muestra asociada con complicaciones graves y potencialmente mortales, si bien no afectó la mortalidad operatoria. Creemos importante que las unidades de cuidados cardiológicos trabajen con protocolos de reconocimiento de pacientes de mayor riesgo y tratamiento de la FAPO.

Este trabajo nos motiva a continuar investigando en las causas y consecuencias de la FAPO, así como en la generación de medidas efectivas para su profilaxis y tratamiento.

Contribución de autores

Jimena Pacella, https://orcid.org/0000-0001-8732-9674 Idea original, participación principal en ejecución, análisis, redacción y respuesta a editores.

Fernanda Molina, https://orcid.org/0000-0003-3664-5647 Recolección de datos.

Andreina Gómez, https://orcid.org/0000-0003-1911-8730 Diseño.

Lucía Florio, https://orcid.org/0000-0002-9608-2666 Diseño, análisis estadístico, supervisión general del trabajo.

Este artículo fue aceptado para su publicación por: Editor jefe Dr. Gerardo Soca.

\section{Bibliografía}

1. Alqahtani AAR. Atrial fibrillation post cardiac surgery trends toward management. Hear Views. 2010; 11(2):57. doi:10.4103/1995-705X.73212

2. Mathew JP, Fontes ML, Tudor IC, Ramsay J, Duke P, Mazer CD, et al. A multicenter risk index for atrial fibrillation after cardiac surgery. JAMA. 2004;291(14):1720-9. doi:10.1001/jama.291.14.1720

3. LaPar DJ, Speir AM, Crosby IK, Kron IL, Speir AM, Fonner E, et al. Postoperative atrial fibrillation significantly increases mortality, hospital readmission, and hospital costs. Ann Thorac Surg. 2014;98(2):527-33; discussion 533. doi:10.1016/j.athoracsur.2014.03.039

4. Saxena A, Dinh DT, Smith JA, Shardey GC, Reid CM, Newcomb AE. Usefulness of postoperative atrial fibrillation as an independent predictor for worse early and late outcomes after isolated coronary artery bypass grafting (multicenter Australian study of 19,497 patients). Am J Cardiol. 2012;109(2):219-25. doi:10.1016/j.amjcard.2011.08.033

5. El-Chami MF, Kilgo P, Thourani V, Lattouf OM, Delurgio DB, Guyton RA, et al. New-onset atrial fibrillation predicts long-term mortality after coronary artery bypass graft. J Am Coll Cardiol. 2010;55(13):1370-6. doi:10.1016/j.jacc.2009.10.058

6. Kowey PR, Stebbins D, Igidbashian L, Goldman SM, Sutter FP, Rials SJ, et al. Clinical outcome of patients who develop PAF after CABG surgery. Pacing Clin Electrophysiol. 2001;24(2):191-3. doi: 10.1046/j.1460-9592.2001.00191.x

7. Ad N, Snir E, Vidne BA, Golomb E. Potential preoperative markers for the risk of developing atrial fibrillation after cardiac surgery. Semin Thorac Cardiovasc Surg. 1999;11(4):308-13. doi: 10.1016/s10430679(99)70074-2

8. Ak K, Akgun S, Tecimer T, Isbir CS, Civelek A, Tekeli A, et al. Determination of histopathologic risk factors for postoperative atrial fibrillation in cardiac surgery. Ann Thorac Surg. 2005;79(6):1970-5. doi:10.1016/j.athoracsur.2004.12.029

9. Mitchell LB; CCS Atrial Fibrillation Guidelines Committee. Canadian Cardiovascular Society atrial fibrillation guidelines 2010: prevention and treatment of atrial fibrillation following cardiac surgery. Can J Cardiol. 2011;27(1):91-7. doi:10.1016/ j.cjca.2010.11.005

10. Arsenault KA, Yusuf AM, Crystal E, et al. Interventions for preventing post-operative atrial fibrillation in patients undergoing heart surgery. Cochrane database Syst Rev. 2013;(1):CD003611. doi:10.1002/ 14651858.CD003611.pub3

11. Goette A, Staack T, Röcken C, Arndt M, Geller JC, Huth C, et al. Increased expression of extracellular signal-regulated kinase and angiotensin-con- 
verting enzyme in human atria during atrial fibrillation. J Am Coll Cardiol. 2000;35(6):1669-77. doi: 10.1016/s0735-1097(00)00611-2

12. Mayson SE, Greenspon AJ, Adams S, Decaro MV, Sheth M, Weitz HH, et al. The changing face of postoperative atrial fibrillation prevention: a review of current medical therapy. Cardiol Rev. 2007;15(5):231-41. doi:10.1097/CRD.0b013e31813e62bb

13. Lee JK, Klein GJ, Krahn AD, Yee R, Zarnke K, Simpson C, et al. Rate-control versus conversion strategy in postoperative atrial fibrillation: trial design and pilot study results. Card Electrophysiol Rev. 2003;7(2):178-84. doi: 10.1023/a:102742800360914.

Gillinov AM, Bagiella E, Moskowitz AJ, Raiten JM, Groh MA, Bowdish ME, et al. Rate control versus rhythm control for atrial fibrillation after cardiac surgery. N Engl J Med. 2016;374(20):1911-21. doi:10.1056/NEJMoa1602002

15. Agewall S, Camm J, Barón Esquivias G, Budts W, Carerj S, Casselman F, et al. Guía ESC 2016 sobre el diagnóstico y tratamiento de la fibrilación auricular, desarrollada en colaboración con la EACTS. Rev Esp Cardiol. 2017;70(1):50.e1-50.e84. doi:10.1016/j.recesp.2016.11.014
16. Martinez EA, Epstein AE, Bass EB; American College of Chest Physicians. Pharmacologic control of ventricular rate: American College of Chest Physicians guidelines for the prevention and management of postoperative atrial fibrillation after cardiac surgery. Chest. 2005;128(Suppl 2):S56-60. doi: 10.1378/chest.128.2_suppl.56s

17. Gialdini G, Nearing K, Bhave PD, Bonuccelli U, Iadecola C, Healey JS, et al. Perioperative atrial fibrillation and the long-term risk of ischemic stroke. JAMA. 2014;312(6):616. doi:10.1001/jama. 2014.9143

18. Thygesen K, Alpert JS, Jaffe AS, Chaitman BR, Bax JJ, Morrow DA, et al. Consenso ESC 2018 sobre la cuarta definición universal del infarto. Rev Esp Cardiol. 2019;72(1):72.e1-72.e27. doi:10.1016/j.recesp.2018.11.011

19. Irigoin J, Zócalo Y, Cortelezzi Z, González V, ReyesW, Varela G, et al. Incidencia de la fibrilación auricular poscirugía de revascularización miocárdica: asociación con uso de circulación extracorpórea. Rev Urug Cardiol. 2007; 22: 123-9. 